\title{
НАРУШЕНИЯ МОЧЕИСПУСКАНИЯ И СОЧЕТАННЫЕ НАРУШЕНИЯ ФУНКЦИИ ТАЗОВЫХ ОРГАНОВ У ДЕТЕЙ: ПОДХОДЫ К ДИАГНОСТИКЕ, ЛЕЧЕНИЮ И ПРОФИЛАКТИКЕ
}

\author{
А. Б. Моисеев, А. А. Миронов ${ }^{凶}$ О. Б. Кольбе, Е. Е. Вартапетова, В. В. Полунина, А. А. Аль-Сабунчи, В. С. Полунин,
}

Г. Н. Буслаева

Педиатрический факультет, Российский национальный исследовательский медицинский университет имени Н. И. Пирогова, Москва

Нарушения мочеиспускания у детей, проявляющиеся недержанием мочи, нестерпимыми позывами к микции и т. д., являются на сегодняшний день одной из актуальных проблем отечественного здравоохранения. Несмотря на широкое освещение данной проблематики в отечественныхи зарубежных источниках литературы, у врачей особенно первичного звена здравоохранения регулярно возникают сложности в правильной диагностике расстройств мочеиспускания, в том числе энуреза. По результатам ретроспективного анализа расхождения направительных диагнозов (из первичного звена здравоохранения) и заключительных клинических диагнозов у детей с нарушениями мочеиспускания даны рекомендации по правильной диагностике расстройств мочеиспускания, а также сочетанных нарушений функции тазовых органов; предложен алгоритм диагностики данных нарушений.

Ключевые слова: дети, нарушения мочеиспускания, недержание мочи, энурез, энкопрез, диагностика и лечение, трудности диагностики

$\triangle$ Для корреспонденции: Андрей Анатольевич Миронов ул. Островитянова, д. 1, г. Москва, 117997; 7190382@mail.ru

Статья получена: 19.06.18 Статья принята к печати: 26.11.18

DOI: $10.24075 /$ vrgmu.2018.067

\section{URINARY DISORDERS AND BLADDER-BOWEL DYSFUNCTION IN CHILDREN: APPROACHES TO DIAGNOSIS, TREATMENT AND PREVENTION}

Moiseev AB, Mironov AA ${ }^{凶}$, Kolbe OB, Vartapetova EE, Polunina W, Al-Sabunchi AA, Polunin VS, Buslaeva GN

Faculty of Pediatrics, Pirogov Russian National Research Medical University, Moscow

Pediatric urinary disorders manifested as urinary incontinence, compelling urges to urinate, etc. remain an important problem of contemporary healthcare. In spite of being extensively covered in the literature, urinary disorders, including enuresis, still present a diagnostic challenge to the physicians of primary healthcare facilities. Based on the findings of our retrospective study that revealed discrepancies between referral and final diagnoses of pediatric urinary disorders, we give recommendations to both physicians of primary healthcare facilities and their inpatient care colleagues that will help them to arrive at the correct diagnosis of a urinary disorder or concomitant bladder-bowel dysfunction using the proposed diagnostic algorithm.

Keywords: children, urinary disorders, urinary incontinence, enuresis, encopresis, diagnosis and treatment, diagnostic difficulties

Correspondence should be addressed: Andrey A. Mironov

Ostrovityanova 1, Moscow, 117997; 7190382@mail.ru

Recieved: 19.06.18 Accepted: 26.11.18

DOI: $10.24075 /$ brsmu.2018.067

В течение последних 25-30 лет одной из актуальных проблем педиатрии, несрологии и урологии являются лечение и реабилитация детей с различными проявлениями нарушения мочеиспускания (МИ). Среди заболеваний органов мочевыделительной системы (МВC), связанных с нарушениями уродинамики нижних отделов мочевых путей, расстройства МИ представляют собой наиболее распространенные и разнообразные по форме нарушения резервуарной и эвакуаторной функции мочевого пузыря (МП) [1, 3-6].

\section{Этиология, патофизиология}

На формирование нарушений МИ оказывают влияние различные факторы, ведущими из которых являются стрессовые воздействия, нарушения иннервации, неврозы и неврозоподобные состояния, резко изменяющиеся условия психосоциального окружения человека, наследственная предрасположенность, инфекционновоспалительные заболевания органов МВС. Кроме того, в последнее время акцентируется внимание на психосоматических аспектах нарушений МИ [2, 7-10].

Мочеиспускание представляет собой сложный периодически наступающий реслекторный акт выделения определенного количества мочи по уретре из МП, является конечным этапом транспорта мочи из организма. У новорожденных и грудных детей МИ осуществляется рефрлекторно, без контроля сознания [4, 11, 12]. Нередко нарушения МИ оказываются следствием задержки формирования «зрелого типа мочеиспускания» и позднего освоения ребенком навыков пользования туалетом. Сознательный контроль МИ днем появляется у 
детей к 2-3 годам и у большинства закрепляется в возрасте 3-4 лет, что связано с возрастными особенностями созревания центров регуляции в головном и спинном мозге, миелинизацией периферических нервных волокон. K 4-5 годам достигается контроль над МП ночью, при этом у мальчиков он формируется позднее, чем у девочек. Контроль МИ днем и ночью позволяет ребенку управлять функциями своего тела, а также обеспечивает его адекватную социализацию. Однако о «зрелом типе мочеиспускания» можно говорить, когда у ребенка сформировалось волевое управление сфинктерным аппаратом МП: он полностью удерживает мочу в МП днем и ночью, умеет задерживать и прерывать при необходимости акт МИ [3, 4, 8, 13].

На формирование «зрелого типа мочеиспускания» влияет и поведение родителей, которые с 15-18 месяцев жизни ребенка должны начинать активно вырабатывать у него навыки чистоплотности, опрятности, умение пользоваться горшком, а затем унитазом. Таким образом, родители дают понять ребенку, какое поведение в социуме является предпочтительным. Только правильно сформированный и мотивационно подкрепленный родителями навык МИ и дефекации в горшок быстро осваивается ребенком. В этом процессе важно все, включая выбор родителями емкости для осуществления физиологических отправлений ребенка (горшка). Согласно отечественным и зарубежным рекомендациям начинать приучать ребенка к горшку следует в среднем с 18 месяцев [14]. Важно, чтобы горшок имел простую, но удобную для посадки ребенка форму, был изготовлен из сертифицированных и легко моющихся материалов, был однотонным. Необходимо исключать из использования горшки, несущие двойную функцию (горшок-игрушка, горшок-стульчик и т. д.), окрашенные в многочисленные яркие цвета. C самых первых дней использования горшок у ребенка должен быть связан только с актами МИ и дефекации, а не ассоциироваться с местом игры, сна или приема пищи $[10,15,16]$. В течение нескольких лет ребенок учится регулировать МИ и десекацию, испытывая при этом удовлетворение от осуществления контроля над своим телом. В этот период родителям надо быть тактичными и терпеливыми, обеспечивать психологический комфорт в семье. Установлено, что даже незначительные стрессы, а тем более наказание ребенка за несоблюдение навыков опрятности, в 70-75\% случаев способны привести к развитию недержания мочи (днем и/или ночью), каломазания и/или хронического запора функционального характера [1, 2, 10, 17]. Правильное формирование контроля МИ у детей напрямую зависит от усилий родителей и является одним из ключевых этапов социального, психического и психосексуального развития ребенка [2, 17-19].

В последние 10-15 лет родители детей с недержанием мочи активно используют разнообразные гигиенические средства, которые впитывают жидкость (подгузники, трусики, пеленки и т. д.) $[15,18]$. Родителям эти гигиенические средства очень нравятся, так как они удобны, практичны и обеспечивают комфортное существование днем и/или ночью детей с недержанием мочи. Длительное использование гигиенических средств, впитывающих жидкость (подгузники, трусики, пеленки), у детей, страдающих недержанием мочи, негативно влияет на течение заболевания. Ребенок быстро привыкает к тому, что остается сухим при непроизвольном МИ и совсем перестает контролировать этот процесс. Это в свою очередь накладывает отпечаток на формирующейся личности ребенка, ибо поздняя социализация посредством в том числе освоения туалетных навыков и контроля над своим телом неизбежно приведет $\mathrm{k}$ инфантилизации. Также следует отметить, что, несмотря на внедрение в производство подгузников материалов, пропускающих воздух и предотвращающих перегревание гениталий, отечественные и зарубежные врачи признают, что длительное регулярное использование подгузников может оказать негативное воздействие на мужскую репродуктивную систему в связи с систематическим воздействием на нее повышенных температур [8, 11, 15, 20].

По результатам наших наблюдений и данным литературы для успешной терапии недержания мочи у детей, использующих гигиенические средства, впитывающие жидкость, на первом этапе необходимо постепенно отказаться от их применения. Часто это сделать непросто, так как при детальном изучении данной проблемы выявляется парадокс: подгузник использует ребенок, а психологический комфорт от его применения в большей степени испытывают родители. Нередко родители детей с недержанием мочи выстраивают психологическую защиту, полагая, что если ребенок спит (бодрствует) в подгузнике, а постельное белье (одежда) остаются «сухими", то с ним все в порядке, он «здоров». При этом ребенок продолжает мочиться непроизвольно, не контролируя этот процесс. Убедить же родителей отказаться от применения подгузников бывает гораздо сложнее, нежели самого ребенка отучить от использования впитывающих гигиенических средств [12, 15, 21, 22].

В ряде работ по нейрофизиологии и урологии авторы связывают нарушения МИ и уродинамики с задержкой созревания высших центров вегетативной регуляции, осуществляющих контроль нижних отделов мочевых путей. Немаловажную роль в формировании нарушений МИ исследователи отводят гипоталамо-гипофизарной недостаточности. Нередко у детей с расстройствами МИ отмечаются негрубые нарушения биоэлектрической активности головного мозга, выявляемые при электроэнцефалографии, а также изменения в работе вегетативной нервной системы во время бодрствования (гипертонус и гиперактивность симпатического и парасимпатического отделов). На развитие расстройств МИ оказывают воздействие отклонения в биологическом ритме «сон-бодрствование», связанные с нарушениями процессов регуляции в лимбико-ретикулярном комплексе, а также патологические изменения в различных отделах эндокринной системы (гипофизарно-надпочечниковом, гипосризарно-половом, соматотропинообразующем и др.) $[1,4,8,10]$.

В ряде исследований было доказано, что выраженность нарушения функций МП напрямую зависит от тяжести и характера патологических изменений, протекающих в нервной системе. Важно, что в детском возрасте на фоне правильно подобранной и адекватной терапии функции МП восстанавливаются вместе с нормализацией работы высших центров вегетативной регуляции МИ. Это является ключевым моментом в подборе эффективных методов лечения и реабилитации детей с расстройствами МИ [5, $9,17,23]$.

Установлено, что около 90\% всех нарушений МИ носят функциональный характер, что позволяет врачу эфффективно использовать для их коррекции помимо традиционной лекарственной терапии немедикаментозные методы и ряд психологических методик. В то же время 
темпы регресса нарушений созревания центров регуляции МИ всегда индивидуальны. Поэтому на сегодняшний день для успешного лечения и реабилитации детей с нарушениями МИ требуется разработка схем терапии, обязательно учитывающих индивидуальные особенности организма пациента [1, 11, 24, 25].

Клинические проявления расстройств МИ разнообразны: от различных сочетаний недержания мочи во время и сна и/или бодрствования до всевозможных изменений частотных и объемных характеристик МИ, а также позывов к микции. Наиболее распространены клинический синдром императивного мочеиспускания (СИМ), проявляющийся в виде поллакиурии, императивных позывов, императивного недержания мочи, а также энурез. По данным литературы, частота встречаемости нарушений МИ среди детского населения составляет 10\%, а среди пациентов нефроурологического профиля 50-60\%. Часто расстройства МИ проявляются в виде недержания мочи, которым страдают не только дети, но и от 1-2\% до 5-25\% взрослых $[4,13$, $16,25]$.

Необходимо признать, что до сих пор в обществе присутствует двойственное отношение к МИ и десекации, приводящее к несвоевременной диагностике и запоздалому лечению нарушений в данной сфере. C одной стороны, повсеместно декларируется позиция, что МИ и десекация - это фризиологические акты организма человека (наряду со сном, приемом пищи и т. д.), обеспечивающие его полноценное существование. С другой стороны, МИ и дефекация воспринимаются как «грязные» и «низменные» стороны человеческого бытия, а обсуждение проблем, связанных с нарушением их функций (в том числе с врачом), считается верхом неприличия и невоспитанности. В итоге, МИ и дефекация в сознании людей становятся «изгоями», информация о них в социуме табуирована, а потому проблемы в этой сфере считаются деликатными и неподлежащими разглашению. В результате нарушения МИ и дефекации не осознаются пациентами как патология, потому они «до последнего» откладывают визит к врачу. Вследствие этого и возникает такая разница в цифрах (1-25\%) при проведении рандомизированных исследований по данному вопросу, что связано с этикодеонтологическими и психосоциальными проблемами, так как людям бывает нелегко признаться (даже врачу), что они упускают мочу (и/или кал) с различной степенью выраженности [8, 13, 15, 23].

Энурез - это непроизвольное МИ во время ночного (чаще) и/или дневного (реже) сна, в возрасте, когда должен быть достигнут контроль над МП. По данным отечественной литературы, таким возрастом является 5 лет, по зарубежным - 6 лет. По результатам ряда работ, распространенность энуреза у детей в возрасте от 5-6 до 15 лет составляет 2,3-30\%, среди них в 5 лет - 15-20\% детей, в 6-8 лет - 7-12\% , в 15-18 лет - 1,5-4\%. Если ранее (в том числе по МКБ-10) выделяли первичный энурез, когда у ребенка не было периода контроля над МП, и вторичный, когда такой период был, то сейчас акцентируют внимание на характере течения энуреза [1, 4, 19, 26]. В настоящее время, согласно рекомендациям Международного общества по удержанию мочи у детей (International Children's Continence Society, ICCS; 2011), с клинических позиций энурез разделяют на моносимптомный (моносимптоматический) и немоносимптомный (немоносимптоматический, полисимптомный, полисимптоматический). Для моносимптомного энуреза характерно прерывистое недержание мочи во время сна, отсутствие дополнительных симптомов расстройств МИ во время бодрствования, как в фазу накопления мочи в МП, так и в фазу его опорожнения. Под немоносимптомным энурезом понимают состояние, при котором кроме энуреза отмечается различная симптоматика нарушений МИ днем. Под термином «дневное недержание мочи» понимают нарушения, происходящие во время бодрствования (без наличия факта недержания мочи во время сна). Сведения о частоте встречаемости моносимптомного и немоносимптомного энуреза у детей различаются. Согласно одним данным, моносимптомный энурез встречается у 43\% детей, а немоносимптомный у 57\%, согласно другим, моносимптомный энурез выявляется у 68,5\% пациентов, а немоносимптомный у $31,5 \%$ [3, 7, 8, 11]. По результатам наших наблюдений, моносимптомный энурез отмечается у $24 \%$ детей, немоносимптомный энурез - у 58\%, а дневное недержание мочи - у 18\% пациентов.

Волнение и стрессовые ситуации часто сопровождаются учащенными МИ, которые могут спровоцировать возникновение недержания мочи или усугубить уже имеющиеся явления недержания. Однако, если энурез, возникающий во время сна (ночью), чаще скрыт от посторонних глаз (за исключением детей, находящихся В коллективах или организациях с круглосуточным пребыванием, гостях и т. д.), то непроизвольное МИ днем нередко случается в общественных местах. Публичное непроизвольное МИ во время бодрствования наносит ребенку более серьезную психологическую травму, нежели энурез [2, 10, 18, 25].

Таким образом, на сегодняшний день проблема расстройств МИ стоит очень остро. Это связано с особенностями восприятия ее в обществе, в семье, а также с недостаточной медицинской грамотностью населения. В большинстве случаев к нарушениям МИ относятся не как к заболеваниям, требующим обследования и лечения ребенка, а как к постыдному в социально-гигиеническом плане явлению, отсутствию элементарного воспитания, навыков опрятности и даже как к элементу умственной отсталости. В семье в силу сложившихся стереотипов проблему нарушения МИ порой замалчивают или не замечают вовсе, относятся к ней как к проявлению «особенностей» ребенка, наказывают детей, ждут пока они ее «перерастут» и не обращаются к врачу [15, 20, 21].

Расстройства МИ (например, недержание мочи, поллакиурия, изменение характеристик позыва к микции) непосредственно не угрожают жизни пациента, но являются социально значимой проблемой. Они приводят $\mathrm{k}$ выраженному ограничению психической и физической активности ребенка, вызывают чувство вины, ущербности, тем самым оказывают негативное влияние на психологическое развитие его личности, снижают адаптацию в обществе и качество жизни всей семьи [8, $22,25]$. В результате возникают проблемы в общении со сверстниками, отставание в учебе, конфрликтные ситуации в семье. Необходимо отметить, что в 30\% случаев расстройства МИ могут привести к развитию рецидивирующего хронического цистита, пузырномочеточникового рефлюкса, уретерогидронефроза, пиелонефрита с возникновением в дальнейшем нефросклероза и ренальной артериальной гипертензии. Нарушение кровообращения и застойные явления в сосудах органов малого таза, а также слабость мышц тазового дна являются частыми спутниками нарушений МИ и сочетанных нарушений функции тазовых органов (СНФТО). Поэтому у взрослых эти заболевания могут 
привести к формированию разнообразных дисфункций со стороны половой сферы у мужчин (импотенция, преждевременная эякуляция и бесплодие) и женщин (аноргазмия, вагинизм, выпадение слизистой оболочки гениталий и невынашивание беременности). Таким образом, формируясь в детстве, нарушения МИ и СНФТО В конечном счете приводят к ранней инвалидизации экономически активных групп граждан и резко ухудшают качество жизни людей $[8,23,27]$.

\section{Сложности диагностики и выбора терапии нарушений МИ и дефекации у детей}

В ходе наблюдения и обследования большого числа пациентов с нарушениями МИ нами был замечен ряд негативных тенденций в первичном звене здравоохранения (в большей степени организационного характера), связанных с несвоевременной и неадекватной диагностикой расстройств МИ и сопутствующих им заболеваний тазового дна у детей.

За последние 20 лет за рубежом, а затем и в Российской Федерации (РФ) глобальным образом изменились подходы к диагностике, лечению и реабилитации пациентов с функциональными нарушениями МИ и СНФТО: лекарственная терапия уступила место немедикаментозным методикам, все чаще используется комбинация различных методов лечения. На сегодняшний день терапевтические возможности российского здравоохранения не уступают зарубежным. В арсенале отечественных врачей присутствует широкий спектр способов коррекции расстройств МИ и СНФТО: медикаментозная терапия (чаще М-холинолитики), паллиативно-симптоматические операции (у детей применяются крайне ограниченно), физиотерапия, иглорефлексотерапия и психотерапия (методики внушения и самовнушения), уротерапия, а также терапия с применением метода функционального биологического управления (ФБУ). Однако каждый из этих способов лечения, имея определенную эффективность, в РФ претендует на лидерство, поэтому отечественные врачи могут столкнуться со сложностями в подборе терапии, необходимой пациенту. В настоящее время первичная терапия нарушений МИ и дефекации включает в себя немедикаментозные и неинвазивные методы лечения, они завоевали большую популярность среди врачей и пациентов, так как сочетают диагностические и лечебнореабилитационные возможности. К ним, например, относится ФБУ-терапия в режиме электромиографии (БОС-терапия). Это система упражнений для мышц тазового дна (по А. Кегелю), выполняемая под контролем Таблица 1. Распределение проявления СНФТО аппаратно-компьютерного комплекса [16, 28-30]. К немедикаментозному лечению также относят уротерапию, режим спланированных МИ и дефекаций, психотерапию и применение мочевых будильников (alarm-терапия). При неэффективности немедикаментозных методик, описанных выше, переходят к медикаментозному лечению и физиотерапии, а также к сочетанию немедикаментозных методик и лекарственной терапии $[1,8,24,26]$.

Лечение и реабилитация пациентов с расстройствами МИ будут неэффективны, если предшествующая им диагностика заболевания была проведена плохо, поэтому на проблеме выявления нарушений МИ и дефекации необходимо остановиться отдельно. Дистальные отделы МВC и желудочно-кишечного тракта (ЖKT) связаны диафрагмой тазового дна, имеют общие пути кровоснабжения, иннервации и выполняют сходные функции: накопления, удержания и эвакуации содержимого (мочи, кала). Расстройства дистальных отделов мочевого тракта (недержание мочи во время сна и/или бодрствования, СИМ и т. д.) и системы органов пищеварения (хронический запор и/или каломазание), протекая совместно, оказывают друг на друга отрицательное воздействие [8, 20, 23, 27]. При комбинации расстройств функции дистальных отделов MBC и ЖКТ в зарубежных странах наиболее часто используется диагноз «сочетанные нарушения функции тазовых органов» (СНФТО). В отечественной медицинской документации увидеть данную нозологию можно нечасто. Переход зарубежных коллег от так называемого системно-органного способа диагностики заболеваний (по органам одной системы, например МBC или ЖКТ), или узкопрофильного (В зависимости от специализации врача), к функциональному, когда исследуются совместно функционирующие области организма, объединяющие несколько различных систем, на наш взгляд, является важнейшей предпосылкой к успешной диагностике и лечению СНФТО. Подобный междисциплинарный подход позволяет врачам различных специальностей (педиатрам, нефрологам, урологам, гастроэнтерологам, проктологам, неврологам и т. д.) широко смотреть на данную проблему, а главное независимо друг от друга своевременно и правильно диагностировать СНФТО, более тщательно подбирать лекарственные препараты и способы лечения, обладающие однонаправленным действием на дисфункции дистальных отделов МBC и ЖКТ [23, 27].

Вышеизложенное подтверждают данные, полученные нами при обследовании и лечении в 2003-2013 гг. 2043 детей 7-18 лет (881 мальчик и 1162 девочки, средний возраст 9,8 \pm 3,4 года, $p=0,05)$, с нарушениями МИ

\begin{tabular}{|c|c|c|c|c|c|c|}
\hline \multirow{2}{*}{ Проявление СНФТО } & \multicolumn{2}{|c|}{ Мальчики } & \multicolumn{2}{|c|}{ Девочки } & \multicolumn{2}{|l|}{ Всего } \\
\hline & Абс. число & $\%$ & Абс. число & $\%$ & Абс. число & $\%$ \\
\hline Энурез + СИМ + хронический запор + каломазание (энкопрез) & 93 & 4,6 & 161 & 7,8 & 254 & 12,4 \\
\hline Энурез + хронический запор + каломазание & 147 & 7,2 & 53 & 2,6 & 200 & 9,8 \\
\hline Энурез + СИМ + каломазание & 53 & 2,6 & 67 & 3,3 & 120 & 5,9 \\
\hline Энурез + каломазание & 40 & 1,9 & 14 & 0,7 & 54 & 2,6 \\
\hline Энурез + хронический запор & 27 & 1,3 & 12 & 0,6 & 39 & 1,9 \\
\hline Энурез + СИМ + хронический запор & 12 & 0,6 & 15 & 0,7 & 27 & 1,3 \\
\hline СИМ + хронический запор + каломазание & 28 & 1,4 & 25 & 1,2 & 53 & 2,6 \\
\hline СИМ + хронический запор & 3 & 0,1 & 49 & 2,4 & 52 & 2,5 \\
\hline СИМ + каломазание & 13 & 0,6 & 28 & 1,4 & 41 & 2 \\
\hline Итого & 416 & 20,4 & 424 & 20,7 & 840 & 41,1 \\
\hline
\end{tabular}


неорганического генеза. Неорганический (функциональный) характер нарушений мочеиспускания у пациентов был доказан с помощью комплексного нефро-урологического обследования. Выборка обследованных детей состояла из трех групп: 7-10 лет, 11-14 лет и 15-18 лет, каждая из которых была разделена на мальчиков и девочек. Так, у 840 человек (41,1\%), из которых 416 мальчиков (20,4\%) и 424 девочки (20,7\%), помимо имеющихся расстройств функции мочевого пузыря СНФТО протекали в виде различных комбинаций энуреза, СИМ, стрессового недержания мочи, хронического запора и каломазания (энкопреза) (табл. 1).

Среди СНФТО наиболее часто отмечали энурез (34\%), хронический запор (30,6\%), СИМ (26,8\%), а также комбинацию хронического запора и каломазания (24,8\%).

Не менее интересны данные, свидетельствующие об особенностях течения СНФТО в зависимости от возраста. Результаты обследования детей позволяют сделать вывод, что СНФТО в виде комбинации: 1) энуреза, СИМ и каломазания чаще выявляются у мальчиков и девочек в возрасте 7-10 лет и только у девочек 11-14 и 15-18 лет; 2) энуреза, СИМ, каломазания и хронического запора встречаются во всех возрастах, за исключением мальчиков 15-18 лет; 3) энуреза, СИМ и хронического запора - отмечаются только у детей 7-10 лет, а энуреза и каломазания - исключительно у детей 11-14 лет; 4) энуреза, каломазания и хронического запора диагностируются у детей 7-10 лет и у мальчиков 11-14 лет; 5) СИМ и каломазания - выявляются у детей 7-10 лет и девочек 11-14 лет; 6) СИМ и хронического запора отмечаются во всех возрастных группах, но только у девочек, а СИМ, каломазания и хронического запора имеют место только у мальчиков 7-10 лет и у девочек 11-14 лет. При этом, чем старше становятся дети, тем менее разнообразными и реже диагностируемыми будут проявления СНФТО.

В ходе ретроспективного анализа историй болезни детей выявлены расхождения между диагнозами лечебнопрофилактических учреждений (ЛПУ), направлявших детей в стационар (направительный диагноз), и заключительными клиническими диагнозами (табл. 2).

Направительный диагноз «Энурез» выставляли в 2,2 раза чаще на этапе первичного звена здравоохранения у 49,7\% детей, по сравнению с 23\% пациентов, у которых он был верифицирован после стационарного обследования ( $p<0,01)$. Врачами поликлиник у детей часто не выявлялись признаки других синдромов нарушения МИ, а также расстройств дефекации. В результате на момент поступления в стационар у части детей диагнозы не были верифицированы - нейрогенные дисфункции МП неуточненной этиологии отмечали у 20,9\% пациентов. СИМ как самостоятельную нозологическую единицу не указывали в направительных диагнозах ЛПУ. При этом в стационаре изолированный СИМ был диагностирован у 188 человек (9\%). СНФТО на догоспитальном этапе выявляли в 1,4 раза реже $(29,4 \%)$, чем в больнице $(41,1 \%)(p<0,01)$. Таким образом, полученные данные свидетельствуют о том, что на уровне первичного звена здравоохранения отмечается тенденция к гипердиагностике энуреза и недооценке проявлений СИМ и СНФТО в структуре расстройств МИ и дефекации у детей. K тому же до сих пор среди пациентов и, к сожалению, даже врачей бытует заблуждение, что любое нарушение МИ или любое недержание мочи - это энурез. Однако, согласно данным отечественных авторов и рекомендациям ICCS (2011), энурез представляет собой непроизвольное МИ только во время сна. В результате диагноз “энурез», установленный ЛПУ первичного звена здравоохранения, скрывал под собой различные нарушения МИ, протекавшие как изолированно, так и в сочетании с расстройствами дефекации, что закономерно приводило к неадекватным и недостаточным лечебно-диагностическим мероприятиям.

По нашему мнению, причинами гипо- и гипердиагностики, установления неправильных диагнозов и т. д. являются недостаточные знания врачей первичного звена по выявлению расстройств МИ и СНФТО, а также десицит времени у медперсонала для подробного сбора анамнеза и адекватной оценки проблем пациентов. В результате пациенты посещают одного специалиста за другим, правильный диагноз не устанавливается и необходимое своевременное лечение не назначается. $\mathrm{K}$ сожалению, не редки случаи неправильной диагностики расстройств МИ у детей и необоснованного назначения терапии. Следствием этого является нарастающее недовольство родителей и пациентов качеством оказываемой медицинской помощи.

Для исправления сложившейся ситуации необходим регулярный мониторинг знаний врачей (в том числе первичного звена здравоохранения) о нарушениях МИ и дефекации у детей, включение в тематику курсов по подготовке и переподготовке кадров вопросов по диагностике и лечению расстройств МИ и СНФТО у детского населения. В настоящее время работа ЛПУ (в том числе первичного звена здравоохранения) ориентирована на повышение доступности и качества оказания медицинской помощи. Повышение качества жизни детей, в том числе за счет своевременной диагностики нарушений МИ и дефекации, является ведущей задачей для ЛПУ, что позволит им эффективно функционировать в современных непростых финансовоэкономических условиях. Особенно это актуально сегодня, когда свобода выбора пациентами ЛПУ и персонисикация финансовых средств (в том числе в системе

Таблица 2. Распределение направительных и заключительных диагнозов

\begin{tabular}{|c|c|c|c|c|}
\hline \multirow{2}{*}{ Диагноз } & \multicolumn{2}{|c|}{ Направительный } & \multicolumn{2}{|c|}{ Заключительный } \\
\hline & Абс. число & $\%$ & Абс. число & $\%$ \\
\hline Энурез (изолированный) & 1015 & 49,7 & 470 & 23 \\
\hline Синдром императивного мочеиспускания (изолированный) & 0 & 0 & 184 & 9 \\
\hline Энурез и синдром императивного мочеиспускания & 0 & 0 & 268 & 13,1 \\
\hline Энурез и стрессовое недержание мочи & 0 & 0 & 198 & 9,7 \\
\hline Редкие микции & 0 & 0 & 83 & 4,1 \\
\hline Сочетанные нарушения функции тазовых органов & 601 & 29,4 & 840 & 41,1 \\
\hline Нейрогенные дисфункции мочевого пузыря неуточненной этиологии & 427 & 20,9 & 0 & 0 \\
\hline Всего & 2043 & 100 & 2043 & 100 \\
\hline
\end{tabular}




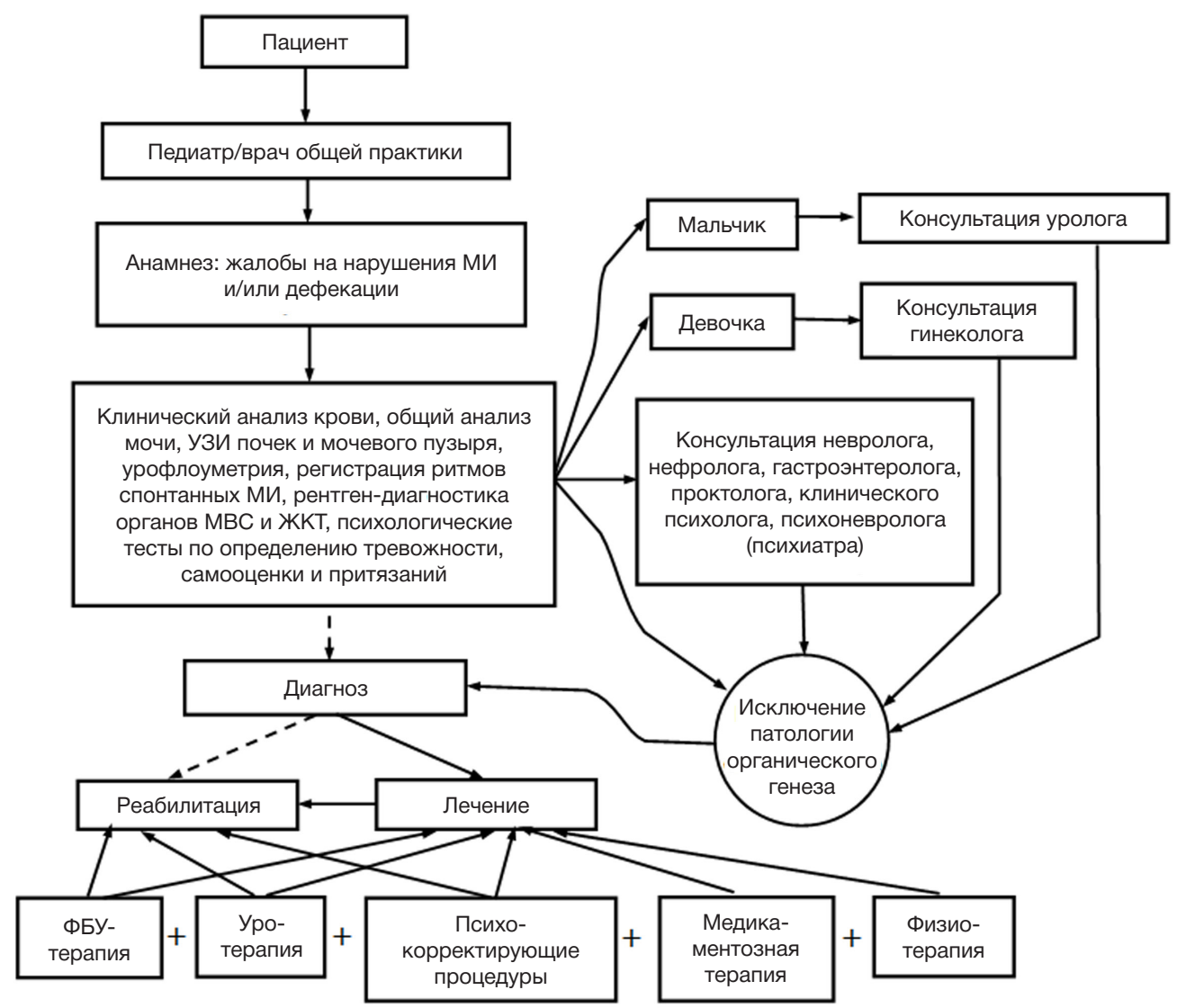

Рис. 1. Схема-алгоритм диагностики расстройств МИ и СНФТО у детей. УзИ - ультразвуковое исследование, ФБУ-терапия - терапия с использованием методики функционального биологического управления, МBC - мочевыделительная система, ЖКТ — желудочно-кишечный тракт

обязательного медицинского страхования) позволяют конкурировать не только частным и государственным клиникам, но и последним между собой.

В помощь врачам стационаров и первичного звена здравоохранения предложена схема-алгоритм диагностики расстройств МИ и СНФТО у детей (рис. 1).

\section{ВЫВОДЫ}

Расстройства МИ и СНФТО у детей остаются актуальной проблемой отечественного здравоохранения. Причины возникновения нарушений МИ и СНФТО разнообразны, а их диагностика требует особого внимания и мультидисциплинарного подхода. У детей с жалобами на недержание мочи всегда следует исключать СНФТО, а также органический генез расстройств МИ и дефекации. Для лечения расстройств МИ и СНФТО на первом этапе применяют функциональное биологическое управление в режиме электромиографии (БОС-терапию), уротерапию, режим спланированных МИ и десекаций, психотерапию, в ряде случаев - мочевые будильники (alarm-терапия). При этом у детей с расстройствами МИ и СНФТО во время бодрствования и сна целесообразно постепенное ограничение использования (Вплоть до полного отказа) гигиенических средств, впитывающих жидкость (подгузники, трусики, пеленки). При неэффективности немедикаментозных методик переходят к медикаментозному лечению и фризиотерапии, а также к сочетанию немедикаментозных методик и лекарственной терапии. Эффективность обследования, лечения и реабилитации пациентов с расстройствами МИ и СНФТО значительно повышается при сохранении преемственности между амбулаторно-поликлинической сетью и стационарами. Это ведет к значительной экономии времени пациентов и врачей, оптимизации использования материально-финансовых и человеческих ресурсов ЛПУ. Для решения проблемы своевременной диагностики, лечения и реабилитации детей с расстройствами МИ и СНФТО на уровне управления здравоохранением применим отечественный и зарубежный опыт, включающий: 1) повышение профессиональной подготовки врачей; 2) увеличение времени приема пациента; 3) расширение диагностической базы ЛПУ и коечной базы отделений дневного пребывания; 4) объединение поликлиник и больниц, а также санаториев в единые амбулаторноклинические и амбулаторно-клинико-санаторные центры по диагностике, лечению и реабилитации пациентов с расстройствами МИ и СНФТО. Вышеуказанные меры могут значительно улучшить качество диагностики, лечения и реабилитации детей с расстройствами МИ и СНФТО. 